\title{
Research on Visual Communication Design under the Influence of Digital Media
}

\author{
Hongwei Nie \\ Lu Xun Art Institute, Yan'an University, Yan'an, Shaanxi, 716000
}

\author{
Keywords: Visual Communication Design, Digital Media, Data Process
}

\begin{abstract}
The main purpose of this study is in the environment of digital information dissemination, visual communication design how to make full use of the excellent characteristics of digital media, based on the traditional design concept, the design language and form of innovation, so that the effective visual information And human transmission, and truly show the digital media in the visual transmission of the formal characteristics. This paper attempts to explore the characteristics of language design, communication characteristics and aesthetic characteristics in the digital media, and use the dynamic form of visual communication in digital media as the starting point, and analyze the basic design elements - the text in the dynamic visual communication In order to provide the reference for the research of other dynamic design fields.
\end{abstract}

\section{Introduction}

Digital information dissemination not only changed people's way of life, but also deeply affected people's thinking. The progress of science and technology led to the emergence of new forms of art, the development of digital technology have changed the work of art design, resulting in a new design concept, and constantly expand the design of the extension. Visual communication design is an important part of contemporary art design, is a combination of art and science and technology integrated disciplines, inevitably affected by the digital age and the impact of the development of technology to bring new opportunities for development, to promote the design concept to Change the way of thinking. Nowadays, the way of visual communication design has been extended from two-dimensional plane to three-dimensional, four-dimensional, communicated by one-way passive transmission toward interactive information exchange development, showing the carrier from the printing media to the digital media field development. The digital age provides a new design language for visual communication design, as well as the traditional design patterns and ideas. Therefore, it must be perfected and integrated in the transformation to find a way to meet the needs of the times. The purpose of visual communication design is to use the aesthetic way to achieve the effective transmission of information, the new era of information with the way to change the way, digital technology and media platform for the design to provide a new display space and design tools, visual communication design Extended to almost all media areas, the design language is reconstructed, the original inherent thinking needs to be constantly fission and derivative. In the digital media environment, the whole face of society has undergone great changes. As a visual communication design of the frontier of information transmission, it is necessary to create works that conform to the needs of the times and adapt to new ways of communication. The development of media technology on the traditional visual communication design of the teaching model and design methods have a huge impact, which is an indisputable fact. In the face of this situation, we should think about how to combine technology and art better, the use of advanced technology for more accurate and effective transmission of information services, while in the course of the study, you can make the designer from a dynamic point of view The use of design forms, in order to achieve the effective transmission of information. 


\section{Visual Communication Design Features}

Digital media visual communication design is mainly through the interface as the carrier to carry out information, the external form of the screen, computer, traditional printing design is based on the form of paper, not to consider the digital media in the interactive, multimedia and Auditory elements, digital interface is the graphic design of digital performance, information into the visual, presented in front of the audience is still graphics, text, color and other visual design elements of the combination of changes in unity, rhythm and rhythm, contrast and balance, repeat , Radiation, deconstruction and other forms of law and form the same applies to the digital media in the visual communication, print design and web interface visual elements of comparison, from the visual analysis, are graphics, text, color design and layout design. Although the same visual design elements, but the digital visual communication design has its special form of expression, is the combination of traditional visual design and modern science and technology.

Visual communication design extends to digital media, media attributes of different, will inevitably lead to new language features, visual language of the virtual, non-materialized design art is the direction of development, interactive, interactive design language so that visual communication More human and humane, and multi-dimensional image space to bring people a new visual experience, these new language is the times and scientific and technological progress to give the design of new features.

Visual communication design is a visual symbol transmission of information activities, it not only includes the production of information design, but also includes the dissemination of information, visual communication design is a visual communication process. Digital age, digital technology and digital media have been deep into people's daily lives, changing the way people accept information. In the digital information dissemination today, media technology, computer technology and visual design of the integration, not only to visual communication design language form has changed, but also changed the way information transmission, from the previous form of static display toward the three-dimensional, dynamic development, communication on the one-way passive interaction, the direction of human extension.

As already mentioned, interactivity is a unique design form of visual communication design in the digital media era. Compared with the traditional print graphic design, the audience has the relative dominance and participation in the process of digital media information dissemination. IT and Internet companies have the function of user research and we can see that the study of the audience's perception of design language and aesthetic psychology of visual communication design has a very important significance.

\section{The Application of Word Design in Dynamic Visual Communication}

Time is the main characteristic of dynamic visual design. From graphic design to dynamic design, the form of font design is transformed from static entity to digital virtual scene based on time and space concept. Time is the sequence that describes the event, and space is the form in which matter is present. In a time-based design, each action or scene is composed of a continuous, time-controlled sequence, forming a timeline, that is, the narrative structure, the dynamic text in the screen is twenty-four frames per second Speed display, through the time series of changes in the static image to produce dynamic, and because of the movement of the text changes, resulting in reading on the transient, through the visual stay in people's minds to form an information impression. So the designer is in the creation of thinking about how to in a short period of time through the movement of the font to carry out effective information transmission, to the people left a deep impression. Although the screen is flat, two-dimensional, but in fact is an all-encompassing virtual space, it covers the content is not limited by the dimension, may be three-dimensional, four-dimensional or even multi-dimensional. In a visual environment, when we look at the perspective of the movement of the object changes, will find the sense of distance from the space, the sense of direction is generated by the physical depth, such as a simple fade out of action, will 
bring us The depth of the visual space, giving people a visual look. In the dynamic design, the development of digital technology and information technology for the text of the spatial properties of the possible, time and space in the movement is an inseparable two concepts, in the space of the transformation of the movement to experience the visual experience, Time flow, so as to better accept the information.

Compared with the static text, the contents of dynamic text design mainly focus on the changes in font action and the creation of a sense of form, in the process of movement changes, there will be many unpredictable possible. Static fonts focus on the design of the form, while the dynamic font is emphasized through the dynamic relationship between the performance of the information in time narrative. The dynamic presentation of the text is controlled by the sequence of frames, which is the beginning and the end of an action, and the speed and rhythm of the movement is controlled by the number and intensity of the intermediate frame. As shown in Figure 4-3, the key frame is the starting node of the motion, and the intermediate frame increases from the dense to the pitch to produce the motion frequency from fast to slow, and vice versa. The movement effect of the text is mainly dominated by the movement speed and the movement direction. The direction of movement of the text depicts the trajectory of the movement and the trajectory of the form is generally divided into straight, curved, parabolic three. In the dynamic design, the speed of movement is an important key to the expression of words, which controls the rhythm of the whole information dissemination, the different speed of the text will bring people different psychological feelings, fast movement will produce tension, vitality, speed, Of the mood; and slow exercise, then bring people to relax, calm, peaceful feeling, through the movement speed and track the adjustment to accurately express information.

\section{Digital Age Visual Communication Design Outlook}

The development and rise of digital media is the inevitable result of the development of modern digital technology, social economy and humanistic environment. Digital media is the rapid development of digital information technology, with modern digital images, video, network technology and the development of emerging media. It incorporates a variety of communication technologies, so that information dissemination can be achieved in a more diverse way. Social and cultural forms progress, but also to promote the rapid development of digital media, digital technology with the ever-changing digital media with the existence and the possibility of growth, economic strength to make it rapid development. Digital technology and digital multimedia design and other new high-tech integrated means, so that the traditional visual communication design to break the previous plane, static propaganda effect, with personalized and strong visual stimulation effect, fast and varied to pass all kinds of information. This kind of informationization and digital development has become the inevitable trend of contemporary design and it will completely change people's living habits, value orientation, aesthetic taste, forcing us to establish the correct visual communication design new ideas, new ideas and new methods. Digitalization has become a lingering trend in contemporary design.

With the visual communication design in the digital media in the extensive, design focus began to convey from the experience, more and more concerned about the emotional experience in the design process. The interactive nature of digital media provides the possibility of experiential design, and the design of the design is no longer just visual designers can do, it requires user research, visual design, interactive design and software engineers to collaborate. In 2009, Nokia launched a new E71 initiative to promote its design, Users can use the interactive program on the network to create their own feelings at this time photos, through the phone text input can generate beautiful pictures, so that the audience in the process of experience to experience the design of human care. The noisy colors and text bring people into the listening and communication of the imagination, through the image of the invisible flow of data, whether it is visual or emotional are shocking. These are the new media technology development for the design of the possible, so the visual communication design future route should be multi-disciplinary cross, from other areas continue to achieve the possibility of innovation, make full use of digital media interactive features, 
bring people You can experience the visual environment, to achieve the design of human information, people have never seen the visual atmosphere.

Digital information age, computer technology, information science and the development of the network has changed people's way of life and attitude towards life. For the design, the focus of attention is not only the basic function to meet, but gradually turned to the pursuit of emotional level. From the beginning of the 20th century, the design community has begun to pay attention to the design of human and human, to meet people's psychological needs for the design criteria, and this criterion has been throughout the visual communication design development process. Design of human nature is through the materialized works, to achieve the emotional exchange between people. In the digital technology and the rapid development of virtual reality today, the design of high-tech products so that people marvel at the same time, but also make people have a sense of distance, so the modern visual design more and more concerned about the emotional needs of people. In the digital communication process of visual communication design, the integration of humanistic concept is very necessary, and the design of depth and connotation can go long. Therefore, the development of digital visual design needs the support of humanistic thought.

\section{Conclusion}

In the face of information revolution, digital media with computer and network technology as the core is becoming the mainstream form of information communication, and visual communication design as a visual information transmission medium, in the new media environment, its design field and the form of extension was extended. This paper attempts to visualize the design language from the design form, and put forward the future development trend of visual communication design.

\section{References}

[1] He Fei. "New Media": technology or philosophy [J]. Art observation, 2011 (01)

[2] Fan Yuanyuan. Contemporary visual communication design - the value and significance of interaction [J]. Packaging World, 2010 (05)

[3] Tan Xuhong. When the new media art encounter visual communication design [J]. Literature Review, 2010 (04)

[4] Shang Xiaoming. New context of visual communication design in the background of information age [J]. Packaging Engineering, 2009 (11)

[5] Wu Qiao. Dynamic design - visual communication under new media conditions [J]. Digital Fashion, 2009 (04) 